\title{
Accelerated Testing with Multiple Failure Modes under Several Temperature Conditions
}

\author{
Zongyue Yu, ${ }^{1,2}$ Zhiqian Ren, ${ }^{1,2}$ Junyong Tao, ${ }^{1,2}$ and Xun Chen ${ }^{1,2}$ \\ ${ }^{1}$ Science and Technology on Integrated Logistics Support Laboratory, National University of Defense Technology, \\ Changsha, Hunan 410073, China \\ ${ }^{2}$ College of Mechatronic Engineering and Automation, National University of Defense Technology, Changsha, Hunan 410073, China
}

Correspondence should be addressed to Junyong Tao; taojunyongnudt@126.com

Received 10 June 2014; Revised 9 August 2014; Accepted 16 September 2014; Published 30 September 2014

Academic Editor: Phil Scarf

Copyright (C) 2014 Zongyue Yu et al. This is an open access article distributed under the Creative Commons Attribution License, which permits unrestricted use, distribution, and reproduction in any medium, provided the original work is properly cited.

\begin{abstract}
A complicated device may have multiple failure modes, and some of the failure modes are sensitive to low temperatures. To assess the reliability of a product with multiple failure modes, this paper presents an accelerated testing in which both of the high temperatures and the low temperatures are applied. Firstly, an acceleration model based on the Arrhenius model but accounting for the influence of both the high temperatures and low temperatures is proposed. Accordingly, an accelerated testing plan including both the high temperatures and low temperatures is designed, and a statistical analysis method is developed. The reliability function of the product with multiple failure modes under variable working conditions is given by the proposed statistical analysis method. Finally, a numerical example is studied to illustrate the proposed accelerated testing. The results show that the proposed accelerated testing is rather efficient.
\end{abstract}

\section{Introduction}

With the successive development of engineering and science technology, high reliability devices usually operate for many years under working conditions. Accelerated testing has been proposed as a means to predict the performances for highly reliable products. Product reliability can be obtained by using the accelerated testing techniques, in which the devices are subjected to higher-than-normal stress levels, leading to failure within days or weeks rather than years. By fitting the accelerated failure data to an appropriate model, device reliability under normal use conditions can be estimated $[1,2]$.

The acceleration models and the statistical analysis methods have been the main focus of the studies about accelerated testing. The relationship between the stress and the reliability of device is established by an acceleration model. Common acceleration models include the inverse power law model and the Arrhenius model $[3,4]$. A lot of efforts were made by scholars to develop a new acceleration model. Benavides [5] constructed an acceleration model for step-stress and variable-stress situations. van Dorp and Mazzuchi [6] developed a general Bayes exponential inference model. Khamis and Higgins [7] proposed a model known as $\mathrm{KH}$ model for step-stress ALT, which is based on a time transformation of the exponential model. The purpose of statistical analysis is to predict the reliability of product under working conditions based on the accelerated testing data and the acceleration model. Tang et al. [8] obtained MLE for parameters in a multicensored accelerated testing. Xiong [9] discussed MLE for the exponential step-stress ALT with type II censored. Fard and Li [10, 11] and Balakrishnan et al. [12-14] finished a lot of researches about the statistical analysis method of accelerated testing. Most of work on the accelerated testing method assumed that there is a single cause of failure. However, a complicated device may fail due to several causes. The accelerated testing method with multiple failure modes has been a new focus of the accelerated testing researches. Kim and Bai [15] and Craiu and Lee [16] described the situations in engineering when multiple failure modes occurred. McCool [17] presents a technique for calculating estimate intervals for Weibull parameters of a primary failure mode when 


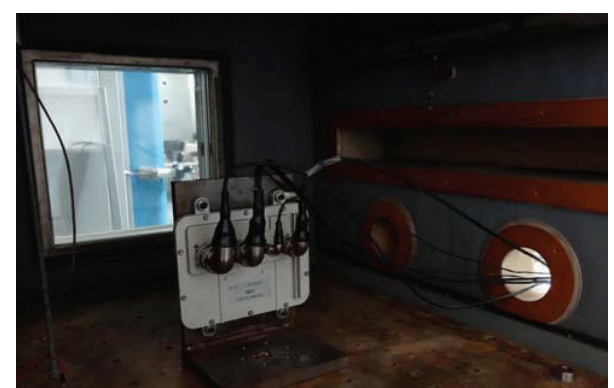

FIGURE 1: The car control device installed in the temperature test chamber.

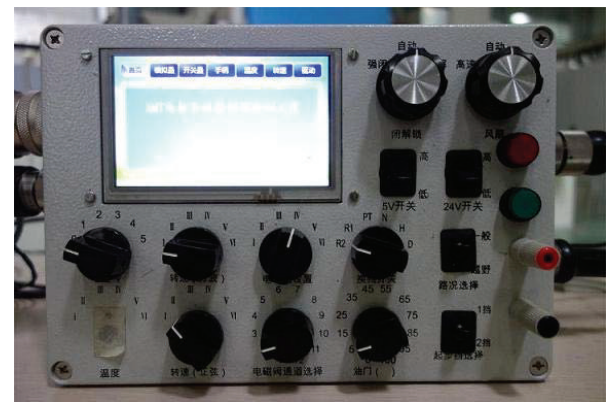

FIGURE 2: The detection equipment for the car control device.

a secondary failure mode having the same Weibull shape parameter is acting. Klein and Basu presented a series of papers $[18,19]$ on the analysis of accelerated testing when more than one failure mode is acting. There are a number of articles on the analysis of multiple failure data, some of which are reviewed in Pascual [20, 21], Liu and Qiu [22], and Xing et al. [23].

High temperatures are widely applied in the existing accelerated testing. Failure modes that are sensitive to high temperatures can be induced quickly by an existing accelerated testing. However, the latest results of the low temperature tests for the car control device and the ship control device described in the next section show that some failure modes are sensitive to the low temperatures. When the high temperature accelerated testing is applied to a product with multiple failure modes, the failure modes that are sensitive to the low temperatures will not be induced and the reliability assessment result will far depart from the actual reliability of the product. To assess the reliability of a product with multiple failure modes, this paper presents an accelerated testing in which not only the high temperatures but also the low temperatures are applied. An acceleration model with multiple failure modes based on the Arrhenius model is given, an accelerated testing plan is designed, and a statistical analysis method is developed. A numerical example shows that the proposed accelerated testing is efficient.

\section{The Low Temperature Tests}

\subsection{The Low Temperature Test for the Car Control Device}

2.1.1. Testing Procedure. The function of the car control device is to receive signals from the control panel and output signals

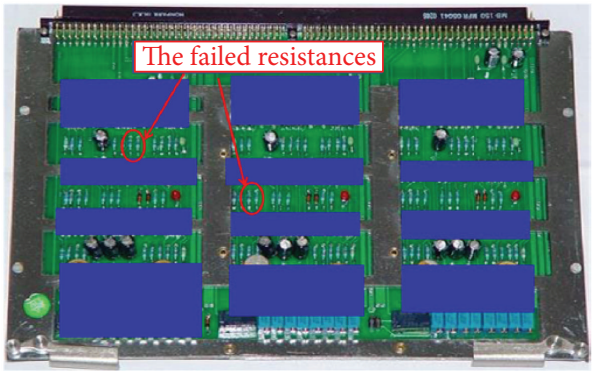

FIgURE 3: The failed resistances.

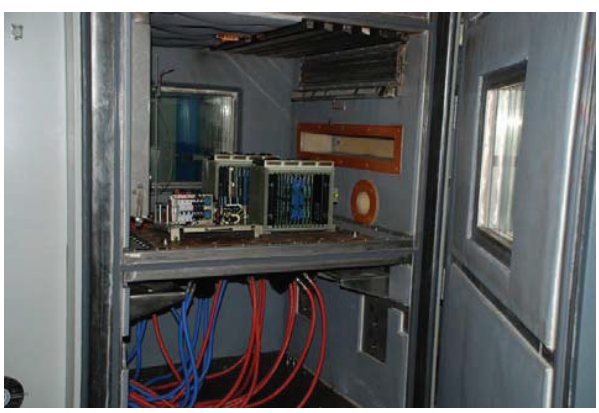

FIGURE 4: The ship control device installed in the temperature test chamber.

to control the speed and direction of the car. In the low temperature test, the car control device is installed in the temperature test chamber (as shown in Figure 1), and the working state of the device is observed by the detection equipment (as shown in Figure 2). At the request of the device producer, the testing temperature is $233 \mathrm{~K}$.

2.1.2. Failure Mode. It is detected that the speed controlled by the device (the output signal is $42 \mathrm{~km} / \mathrm{h}$ ) is lower than the speed set by the control panel $(60 \mathrm{~km} / \mathrm{h})$ when the car control device is tested 220 hours at the temperature $233 \mathrm{~K}$. According to the results of a comprehensive circuit analysis, several resistances values are outside the normal range. The failed resistances are shown in Figure 3 (as the internal structure of the device may be related to the commercial secrets of producer, the parts unrelated to the failure mode are covered).

\subsection{The Low Temperature Test for the Ship Control Device}

2.2.1. Testing Procedure. The ship control device is installed in the temperature test chamber, which is shown in Figure 4. The working state of the device is observed by the detection equipment and shown in Figure 5. At the request of producer, the testing temperature is $223 \mathrm{~K}$.

2.2.2. Failure Mode. It is detected that some working parameters of the device are zero when the ship control device is tested 180 hours at the temperature $223 \mathrm{~K}$. According to the results of a comprehensive circuit analysis, a flip-flop in the communication circuit is out of work.

High temperatures are widely applied in the existing accelerated testing methods. However, the results of the 


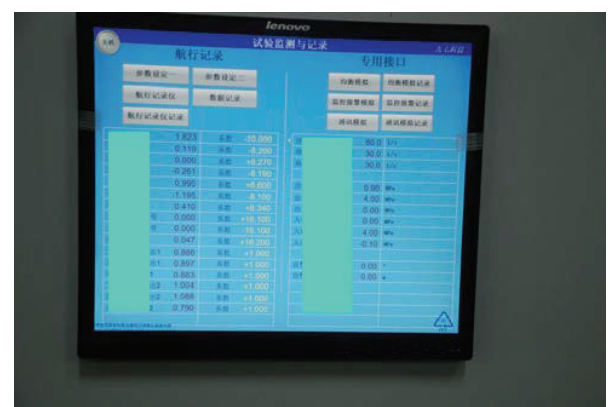

FIGURE 5: The detection equipment for the ship control device.

above low temperature tests show that some failure modes are sensitive to the low temperatures. Obviously, if the existing high temperature accelerated testing is applied to a device with the failure modes that are sensitive to the low temperatures, the reliability estimation result will far deviate from the actual reliability of the device (Figure 6).

\section{Basic Assumptions}

The accelerated testing presented in this paper is based on the following assumptions.

(1) All failure modes are independent of each other.

(2) The failure time of every failure mode is assumed to follow the exponential distribution.

(3) The failure time of every failure mode at different temperatures follows the Arrhenius model.

\section{Acceleration Model}

Arrhenius model was first used by Svante Arrhenius in his studies of the dissociation of electrolytes, but nowadays it is widely accepted as the right tool to describe the influence of temperature on the rates of chemical processes, as well as many other physical processes such as diffusion, thermal and electrical. Arrhenius model can describe the relationship between the temperature and the mean lifetime of products as

$$
\theta(T)=A \exp \left(\frac{E a}{k T}\right)
$$

where $\theta$ is the mean lifetime of products and $A$ is a constant that depends on the product geometry, the specimen size and fabrication, the test method, and other factors. $E_{a}$ is the activation energy of the reaction, usually in electron volts. $k$ is Boltzmann's constant, $8.6171 \times 10^{-5}$ electron volts per ${ }^{\circ} \mathrm{C} . \mathrm{T}$ is the absolute temperature in Kelvin, which is equivalent to the centigrade temperature plus 273.16 degrees.

When the product lifetime follows the exponential distribution, the failure rate of the product is the reciprocal of the mean lifetime. Consider

$$
\lambda(T)=\frac{1}{\theta(T)}=\frac{1}{A} \exp \left(\frac{-E a}{k T}\right)=a \exp \left(\frac{b}{T}\right),
$$

where $a=1 / A, b=-E a / k$.

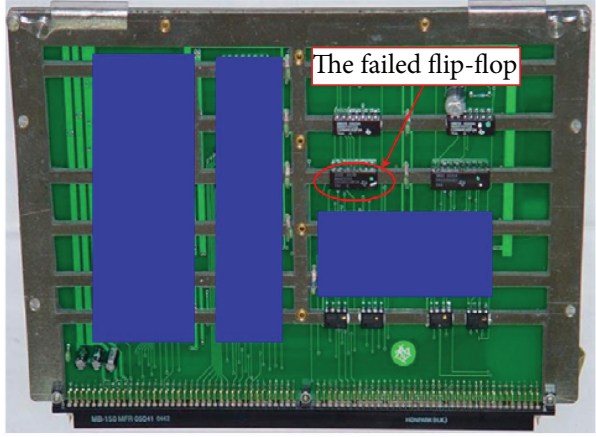

Figure 6: The failed flip-flop.

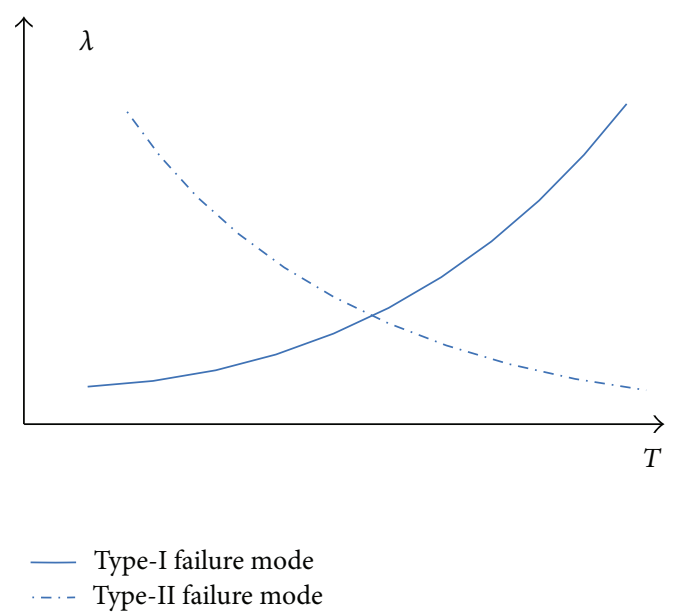

FIgURE 7: Two types of failure mode.

High temperatures are widely applied in the existing accelerated testing methods, and the failure modes (type-I failure mode) that are sensitive to the high temperatures are induced quickly. The failure rate of type-I failure mode grows as the temperature rises, which can be described by the Arrhenius model and shown as the solid line in Figure 7. However, the latest test results of the car control device and the ship control device show that some failure modes (type-II failure mode) are sensitive to low temperatures. The failure rate of type-II failure mode will fall down as the temperature rises, which is shown as the dashed line in Figure 7.

To a complicated device, both the type-I failure mode and type-II failure mode are likely to occur. When the probability that any failure mode occurs is statistically independent, the reliability function of the product can be expressed as

$$
\begin{aligned}
R(t) & =p\left\{\theta_{c}>t\right\} \\
& =p\left\{\min \left\{t_{1}, t_{2} \cdots t_{m}\right\}>t\right\} \\
& =p\left\{\bigcap_{i=1}^{m}\left\{t_{i}>t\right\}\right\}
\end{aligned}
$$




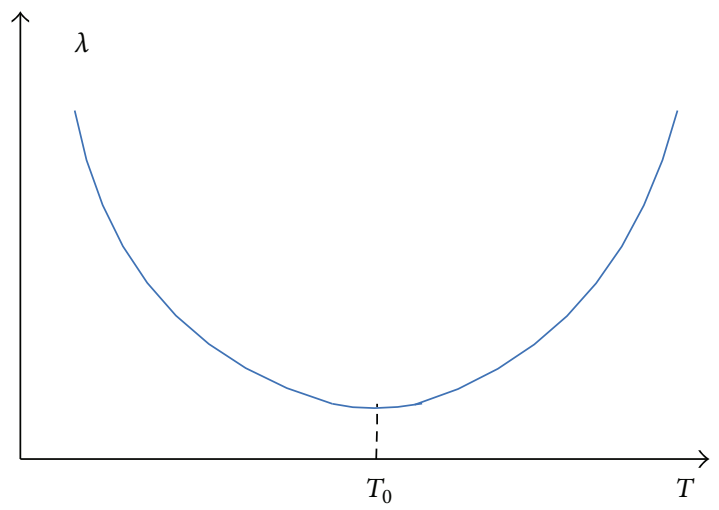

FIGURE 8: The failure rates of multiple failure modes at different temperatures.

$$
\begin{aligned}
& =\prod_{i=1}^{m} p\left\{t_{i}>t\right\} \\
& =\prod_{i=1}^{m} R_{i}(t),
\end{aligned}
$$

where $\theta_{c}$ is the lifetime of the product with multiple failure modes and $t_{1}, t_{2} \cdots t_{m}$ are the failure times of the $m$ failure modes, respectively. $R_{i}(t)$ is the reliability function of the $i$ th failure mode.

According to (3), the product with multiple failure modes can be considered as a series system including $m$ units. The failure rates of the product at different temperatures can be expressed as

$$
\begin{aligned}
\lambda(T) & =\lambda_{h}(T)+\lambda_{l}(T)=\lambda_{1}(T)+\lambda_{2}(T)+\cdots+\lambda_{m}(T) \\
& =a_{1} \exp \left(\frac{b_{1}}{T}\right)+a_{2} \exp \left(\frac{b_{2}}{T}\right)+\cdots+a_{m} \exp \left(\frac{b_{m}}{T}\right) .
\end{aligned}
$$

To a complicated product with multiple failure modes, the type-I failure modes are dominating when the product is under high temperature conditions. Contrarily, the typeII failure modes are primary when the product is under low temperature conditions. Therefore, the failure rates of the device with multiple failure modes at different temperatures can be represented in Figure 8.

\section{Accelerated Testing}

5.1. Design of Accelerated Testing Plan. To estimate the parameters of the Arrhenius model, there should be no less than four levels of temperatures in the accelerated testing plan (the high temperature levels are no less than two and the low temperature levels are no less than two). The type-I censoring testing plan is applied at every temperature level and $t_{0}$ is the censoring time. The test profile is shown as in Figure 9.

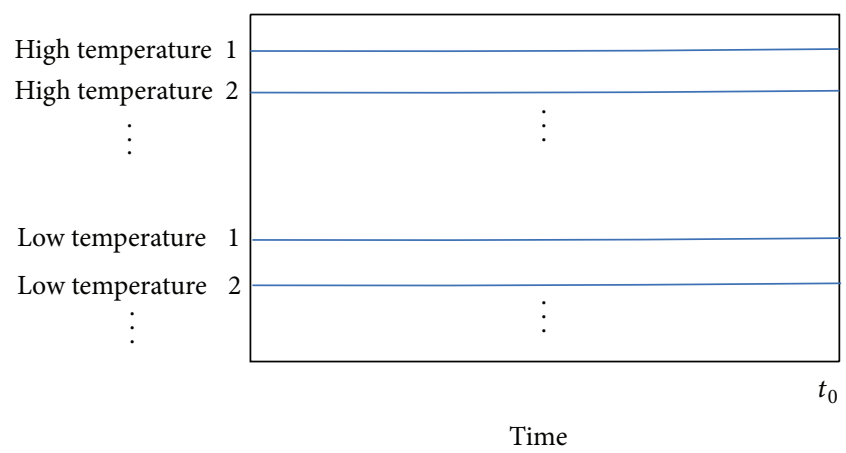

Figure 9: Test profile sketch map.

5.2. Statistical Analysis. The failure time is assumed to follow the exponential distribution when the temperature is a constant. The distribution function of exponential distribution is expressed by

$$
F(t)=1-\exp (-\lambda t)
$$

The failure density function and the reliability function are

$$
\begin{gathered}
f(t)=\lambda \exp (-\lambda t), \\
R(t)=\exp (-\lambda t) .
\end{gathered}
$$

Assuming that there are $m$ failure modes occurring in the test, the $k$ th failure mode is observedin $s_{k}$ samples at times $t_{1}^{(k)}, t_{2}^{(k)} \cdots t_{s_{k}}^{(k)}$. Then, the probability that the $k$ th failure modeis observed in a sample at $t_{j}^{(k)}$ is

$$
p_{j k}=f^{(k)}\left(t_{j}^{(k)}\right)\left[\prod_{i=1}^{m, i \neq j} R^{(i)}\left(t_{j}^{(k)}\right)\right] .
$$

The probability density function of the failure times of all samples can be represented by

$$
\begin{aligned}
L=\prod_{k=1}^{m}\left[\prod_{j=1}^{s_{k}}\left[f^{(k)}\left(t_{j}^{(k)}\right)\left[\prod_{i=1}^{m, i \neq j} R^{(i)}\left(t_{j}^{(k)}\right)\right]\right]\right. \\
\left.\times\left[R^{(k)}\left(t_{0}\right)\right]^{s_{0}}\right],
\end{aligned}
$$

where $\prod_{k=1}^{m}\left[R^{(k)}\left(t_{0}^{(k)}\right)\right]^{s_{0}}$ is the probability that there are $s_{0}$ samples with no failure modes before the censoring time $t_{0}$. 
TABLE 1: Failure times of devices.

\begin{tabular}{lcccc}
\hline \multirow{2}{*}{ Temp. } & Failure 1 & Failure 2 & Failure 3 & Failure 4 \\
\hline $373 \mathrm{~K}$ & $35,43,53,62,73,89$ & $85,96,115,127$ & No sample & No sample \\
$353 \mathrm{~K}$ & $76,83,93,102$, & $162,178,182$ & No sample & No sample \\
$213 \mathrm{~K}$ & No sample & No sample & $106,123,135,139$ & 173,193 \\
$193 \mathrm{~K}$ & No sample & No sample & $18,29,37,45,53,69$ & $78,89,95,97$ \\
\hline
\end{tabular}

TABLE 2: Failure rates of different failure modes at different temperature levels.

\begin{tabular}{lcccc}
\hline Temp. & & Failure rate & & \\
& Failure 1 & Failure 2 & & \\
$373 \mathrm{~K}$ & $7.712 e-003$ & $5.141 e-003$ & & \\
$353 \mathrm{~K}$ & $2.710 e-003$ & $2.033 e-003$ & & \\
$213 \mathrm{~K}$ & & & $2.378 e-003$ & $1.189 e-003$ \\
$193 \mathrm{~K}$ & & & $9.836 e-003$ & $6.557 e-003$ \\
\hline
\end{tabular}

Equation (8) is the likelihood function of all observations. Taking the logarithm on both sides, we have

$\ln L$

$$
\begin{aligned}
& =\ln \left[\prod _ { k = 1 } ^ { m } \left[\prod_{j=1}^{s_{k}}\left[f^{(k)}\left(t_{j}^{(k)}\right)\left[\prod_{i=1}^{m, i \neq j} R^{(i)}\left(t_{j}^{(k)}\right)\right]\right]\right.\right. \\
& \left.\left.\times\left[R^{(k)}\left(t_{0}\right)\right]^{s_{0}}\right]\right] \\
& =\sum_{k=1}^{m}\left[\sum _ { j = 1 } ^ { s _ { k } } \left[\ln \left(\lambda_{k} \exp \left(-\lambda_{k} t_{j}^{(k)}\right)\right)\right.\right. \\
& \left.+\left[\sum_{i=1}^{m, i \neq j} \ln \left(\exp \left(-\lambda_{i} t_{j}^{(k)}\right)\right)\right]\right] \\
& \left.+\ln \left[\left(\exp \left(-\lambda_{k} t_{0}\right)\right)\right]^{s_{0}}\right] \\
& =\sum_{k=1}^{m}\left[s_{k} \ln \lambda_{k}-\lambda_{k}\left[\left[\sum_{j=1}^{s_{k}}\left[t_{j}^{k}\right]\right]+s_{0} t_{0}\right]\right. \\
& \left.+\sum_{j=1}^{s_{k}}\left[\left[\sum_{i=1}^{m, i \neq j}\left(-\lambda_{i} t_{j}^{k}\right)\right]\right]\right] \text {. }
\end{aligned}
$$

The estimation of $\lambda_{k}$ is obtained by maximizing the function (9). Consider

$$
\widehat{\lambda}_{k}=\frac{s_{k}}{\left[\sum_{k=1}^{m} \sum_{j=1}^{s_{k}} t_{j}^{(k)}\right]+s_{o} t_{o}} .
$$

Equation (2) can be rewritten in logarithmic form,

$$
\ln \lambda=\ln a+b \frac{1}{T}
$$

Obviously, $\ln \lambda$ and $1 / T$ are in linear relation. The estimations of Arrhenius model parameters can be obtained by utilizing the least squares method.

Furthermore, the failure rates of a product with multiple failure modes at different temperatures can be acquired by substituting the estimations of Arrhenius model parameters of every failure mode into (4).

In the existing accelerated testing under temperature conditions, the working temperature is usually supposed to be a constant (such as $20^{\circ} \mathrm{C}$ ). However, the working temperatures of the majority of devices are mutative, which can be represented as

$$
T=f(t)
$$

Therefore, the failure rate of product under working environment will also change on time, which can be expressed as

$$
\lambda(T)=g(T)=g(f(t)) .
$$

Finally, the reliability function of device under working conditions can be obtained by

$$
R(t)=e^{-\int_{0}^{t} \lambda(T) d t}=e^{-\int_{0}^{t} g(f(t)) d t}=e^{-E(\lambda) t} .
$$

\section{Illustrative Example}

To apply the proposed accelerated testing to products, a large number of samples will be needed. However, there were insufficient data relating to the car and ship control devices, so instead we present a numerical study where parameter values are motivated by these systems. The products are tested at four temperature levels that are $373 \mathrm{~K}, 353 \mathrm{~K}, 213 \mathrm{~K}$, and $193 \mathrm{~K}$. The sample size at every temperature level is ten and the censoring time is two hundred hours. The failure times of different samples are shown in Table 1.

According to (10), the failure rates of different failure modes at different temperatures are estimated and shown in Table 2. 
TABLE 3: Arrhenius model parameters of different failure modes.

\begin{tabular}{lccrc}
\hline & Failure 1 & Failure 2 & Failure 3 & Failure 4 \\
\hline Parameters & $a_{1}=\exp (13.5939)$ & $a_{2}=\exp (11.1040)$ & $a_{3}=\exp (-19.7425)$ & $a_{4}=\exp (-23.2113)$ \\
estimation & $b_{1}=-6.8852 e+003$ & $b_{2}=-6.1077 e+003$ & $b_{3}=2.9183 e+003$ & $b_{4}=3.5095 e+003$ \\
\hline
\end{tabular}

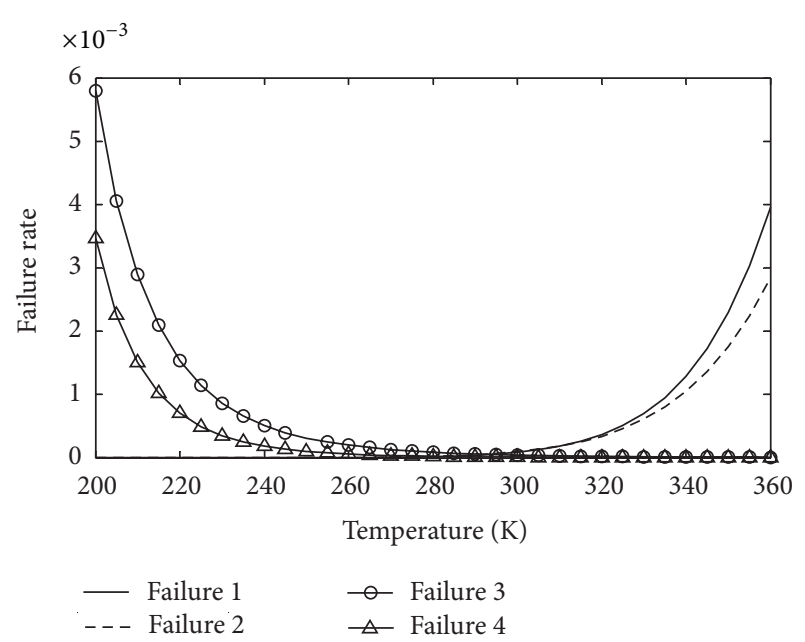

FIGURE 10: Failure rates of different failure modes at different temperatures.

According to (11), the estimations of Arrhenius model parameters are obtained by utilizing the least squares method and represented in Table 3.

The failure rates of different failure modes at different temperatures are calculated by replacing the parameters in (2) with the estimations that are shown in Table 3 . The failure rates of different failure modes at different temperatures are shown in Figure 10.

Furthermore, the failure rates of device with multiple failure modes at different temperatures are acquired by (4). Consider

$$
\begin{aligned}
\lambda= & g(T)=\lambda_{h}+\lambda_{l}=\lambda_{1}+\lambda_{2}+\lambda_{3}+\lambda_{4} \\
= & \exp (13.5939) \times \exp \left(\frac{-6.8852 e+003}{T}\right) \\
& +\exp (11.1040) \times \exp \left(\frac{-6.1077 e+003}{T}\right) \\
& +\exp (-19.7425) \times \exp \left(\frac{2.9182 e+003}{T}\right) \\
& +\exp (-23.2113) \times \exp \left(\frac{3.5095 e+003}{T}\right) .
\end{aligned}
$$

Figure 11 plots the failure rates of product at different temperatures based on (15). The curve denotes the fact that the product is more fail under high temperature environments or low temperature environments. The point indicates the minimal failure rate $(1.5037 e-004)$ when the product is at the temperature of $282 \mathrm{~K}$.

To a complicated device, both of the type-I failure mode and the type-II failure mode are likely to occur. The data

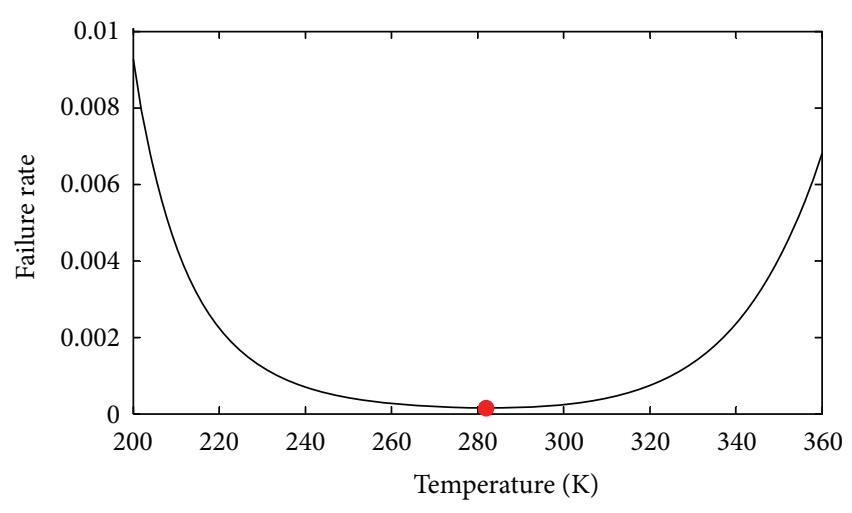

FIGURE 11: Failure rates of the product at different temperatures.

of type-I failure modes and type-II failure modes can be obtained by the proposed accelerated testing. To the existing accelerated testing, only the high temperatures are applied. The type-I failure modes (failure mode 1 and failure mode 2) are induced quickly, but the type-II failure modes rarely occur. Therefore, the failure rate that is estimated by the existing high temperature accelerated testing is

$$
\lambda_{h}=g_{h}(T)=\lambda_{1}+\lambda_{2} .
$$

The estimation of failure rate based on the high temperature accelerated testing is inaccurate due to the neglect of type-II failure modes, and the relative error is defined as

$$
e=\frac{\lambda-\lambda_{h}}{\lambda} \times 100 \%=\frac{\lambda_{3}+\lambda_{4}}{\lambda_{1}+\lambda_{2}+\lambda_{3}+\lambda_{4}} \times 100 \% .
$$

Figure 12 plots the relative error at different temperatures. The curve denotes that the relative error is small under high temperature conditions, while it is large under low temperature conditions. The relative error is less than $10 \%$ when the temperatures are higher than $310 \mathrm{~K}$ and it is more than $90 \%$ when the temperatures are lower than $273 \mathrm{~K}$.

In the existing accelerated testing, the working temperature is usually supposed to be a constant (such as $20^{\circ} \mathrm{C}$ ). However, the working temperatures of the majority of devices are mutative. Figure 13 shows the working temperature of the product. ics is

The expression of the working temperature in mathemat-

$$
T=f(t)= \begin{cases}243 & 0 \leq t<4,20 \leq t<24 \\ 243+20 t & 4 \leq t<8 \\ 323 & 8 \leq t<16 \\ 323-20 t & 16 \leq t<20\end{cases}
$$




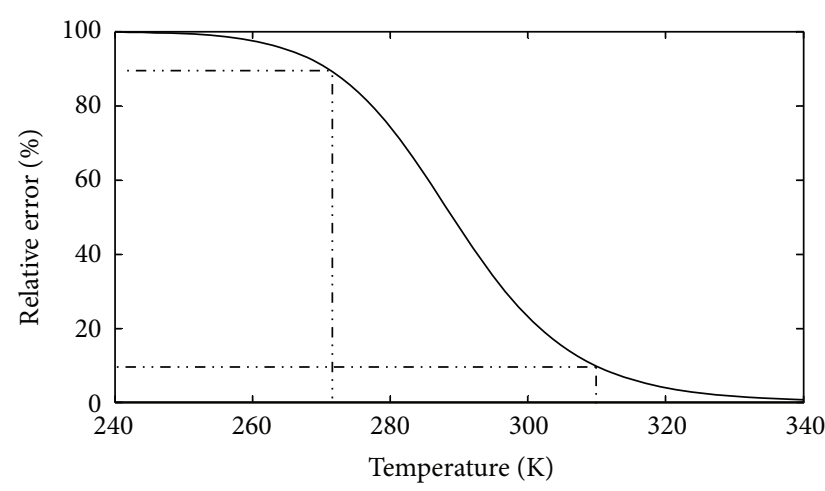

FIGURE 12: Relative error of high temperature accelerated testing.

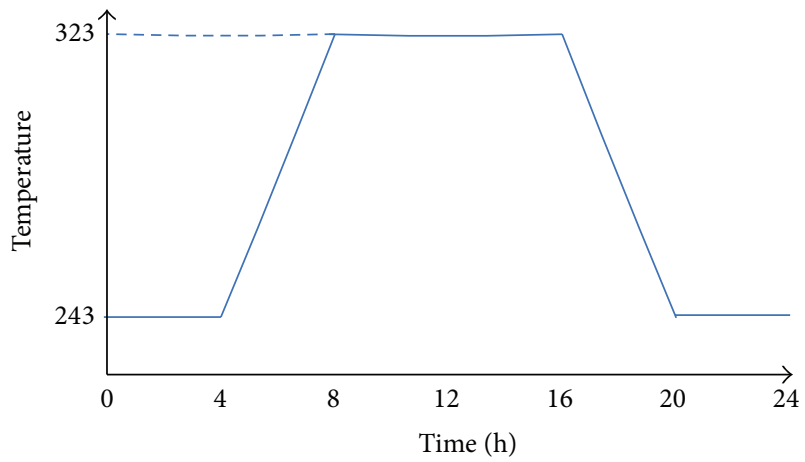

FIgURE 13: The working temperature.

The mean failure rate of all failure modes is calculated by (15) and (18). Consider

$$
E(\lambda)=\frac{\int_{0}^{t} g(f(t)) d t}{t}=\frac{\int_{0}^{24} g(f(t)) d t}{24}=6.0000 e-004 .
$$

The mean failure rate of type-I failure modes is calculated by (16) and (18):

$$
\begin{aligned}
E\left(\lambda_{h}\right) & =\frac{\int_{0}^{t} g_{h}(f(t)) d t}{t}=\frac{\int_{0}^{24} g_{h}(f(t)) d t}{24} \\
& =3.3568 e-004 .
\end{aligned}
$$

According to the mean failure rate of all failure modes, the reliability function of the product obtained by the proposed accelerated testing is

$$
R(t)=\exp (-6.0000 e-004 t) .
$$

According to the mean failure rate of type-I failure modes, the reliability function obtained by the existing high temperature accelerated testing is

$$
R_{h}(t)=\exp (-3.3568 e-004 t) .
$$

The reliability function obtained by the accelerated testing proposed in this paper is shown as the solid line in

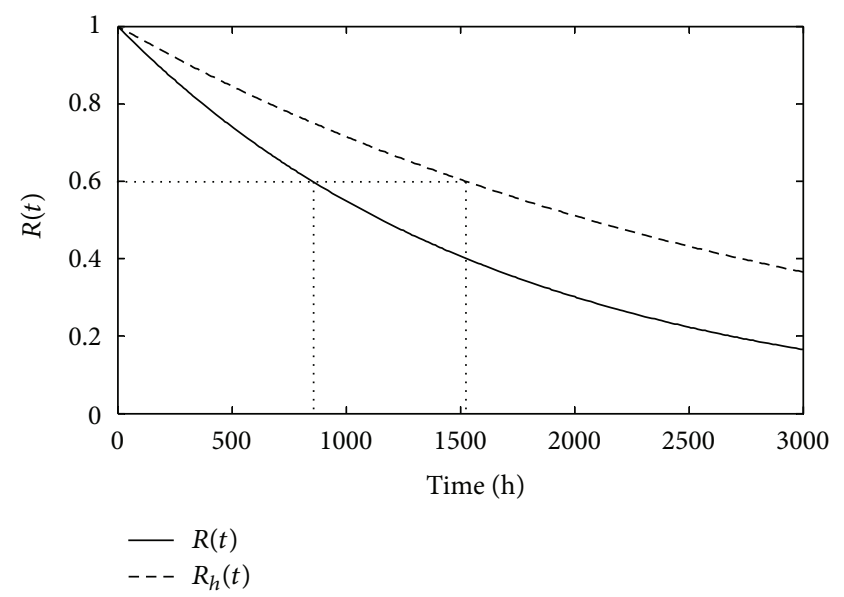

FIGURE 14: Reliability functions obtained by different accelerated testing methods.

Figure 14, while the reliability function attained through the high temperature testing method is shown as the dotted line in Figure 14. The reliability life $\theta_{0.6}$ assessed by the proposed method is 851 hours, while it is assessed by the high temperature testing method is 1521 hour. Obviously, the reliability life is overrated by the high temperature testing method due to the neglect of type-II failure modes.

\section{Conclusion}

By focusing on the reliability assessment of a product with multiple failure modes, this paper presents an accelerated testing in which not only the high temperatures but also the low temperatures are applied. An acceleration model with multiple failures based on the Arrhenius model is given. The corresponding accelerated testing plan is designed, and the statistical analysis method is developed.

The failure rate of a complicated device with both the type-I failure modes and the type-II failure modes can be obtained by the proposed accelerated testing (as shown in Figure 11). The device is more fragile under high temperature conditions or low temperature conditions than the room temperature. The failure rate is minimal $(1.5037 e-004)$ when the product is in the favourable temperature $(282 \mathrm{~K})$.

For the existing accelerated testing, only the high temperatures are applied. The reliability estimation of the high temperature accelerated testing is inaccurate due to the neglect of type-II failure modes (the relative error is shown in Figure 12). In comparison to the high temperature accelerated testing, the proposed accelerated testing could induce both the type-I and type-II failure modes, and the reliability assessed by the proposed accelerated testing is more close to the actual reliability of the product.

\section{Conflict of Interests}

The authors declare that there is no conflict of interests regarding the publication of this paper. 


\section{Acknowledgment}

This study was supported by the National Natural Science Foundation of China (50905181).

\section{References}

[1] M. D. Turner, "A practical application of quantitative accelerated life testing in power systems engineering," IEEE Transactions on Reliability, vol. 59, no. 1, pp. 91-101, 2010.

[2] G. B. Yang, "Accelerated life test plans for predicting warranty cost," IEEE Transactions on Reliability, vol. 59, no. 4, pp. 628634, 2010.

[3] W. Nelson, Accelerated Testing: Statistical Models, Test Plans and Data Analysis, John Wiley \& Sons, New York, NY, USA, 1990.

[4] J. Tao, Z. Yu, Z. Ren, and X. Yi, "Study of an adaptive accelerated model and a data transfer method based on a reliability enhancement test," Eksploatacja i Niezawodność, vol. 16, no. 1, pp. 128-132, 2014.

[5] E. M. Benavides, "Reliability model for step-stress and variablestress situations," IEEE Transactions on Reliability, vol. 60, no. 1, pp. 219-233, 2011.

[6] J. R. van Dorp and T. A. Mazzuchi, "A general Bayes exponential inference model for accelerated life testing," Journal of Statistical Planning and Inference, vol. 119, no. 1, pp. 55-74, 2004.

[7] I. H. Khamis and J. J. Higgins, "A new model for step-stress testing," IEEE Transactions on Reliability, vol. 47, no. 2, pp. 131134, 1998.

[8] L. C. Tang, Y. S. Sun, T. N. Goh, and H. L. Ong, "Analysis of step-stress accelerated-life-test data: a new approach," IEEE Transactions on Reliability, vol. 45, no. 1, pp. 69-74, 1996.

[9] C. Xiong, "Inferences on a simple step-stress model with typeII censored exponential data," IEEE Transactions on Reliability, vol. 47, no. 2, pp. 142-146, 1998.

[10] N. Fard and C. Li, "Optimal simple step stress accelerated life test design for reliability prediction," Journal of Statistical Planning and Inference, vol. 139, no. 5, pp. 1799-1808, 2009.

[11] N. Fard and C. Li, "Optimum bivariate step-stress accelerated life test for censored data," IEEE Transactions on Reliability, vol. 56, no. 1, pp. 77-84, 2007.

[12] N. Balakrishnan and D. Han, "Optimal step-stress testing for progressively Type-I censored data from exponential distribution," Journal of Statistical Planning and Inference, vol. 139, no. 5, pp. 1782-1798, 2009.

[13] N. Balakrishnan, D. Kundu, H. K. T. Ng, and N. Kannan, "Point and interval estimation for a simple step-stress model with typeII censoring," Journal of Quality Technology, vol. 39, no. 1, pp. 35-47, 2007.

[14] N. Balakrishnan, L. Zhang, and Q. Xie, "Inference for a simple step-stress model with type-I censoring and lognormally distributed lifetimes," Communications in Statistics: Theory and Methods, vol. 38, no. 8-10, pp. 1690-1709, 2009.

[15] C. M. Kim and D. S. Bai, "Analyses of accelerated life test data under two failure modes," International Journal of Reliability, Quality and Safety Engineering, vol. 9, no. 2, pp. 111-125, 2002.

[16] R. V. Craiu and T. C. M. Lee, "Model selection for the competing-risks model with and without masking," Technometrics, vol. 47, no. 4, pp. 457-467, 2005.

[17] J. I. McCool, "Competing risk and multiple comparison analysis for bearing fatigue tests," ASLE Transactions, vol. 21, no. 4, pp. 271-284, 1978.
[18] J. P. Klein and A. P. Basu, "Weibull accelerated life tests when there are competing causes of failure," Communications in Statistics A: Theory and Methods, vol. 10, no. 20, pp. 2073-2100, 1981.

[19] J. P. Klein and A. P. Basu, "Accelerated life testing under competing exponential failure distributions," IAPQR Transactions, vol. 7, no. 1, pp. 1-20, 1982.

[20] F. Pascual, "Accelerated life test planning with independent Weibull competing risks," IEEE Transactions on Reliability, vol. 57, no. 3, pp. 435-444, 2008.

[21] F. Pascual, "Accelerated life test planning with independent lognormal competing risks," Journal of Statistical Planning and Inference, vol. 140, no. 4, pp. 1089-1100, 2010.

[22] X. Liu and W. S. Qiu, "Modeling and planning of step-stress accelerated life tests with independent competing risks," IEEE Transactions on Reliability, vol. 60, no. 4, pp. 712-720, 2011.

[23] L. Xing, C. Wang, and G. Levitin, "Competing failure analysis in non-repairable binary systems subject to functional dependence," Journal of Risk and Reliability, vol. 226, no. 4, pp. 406416, 2012. 


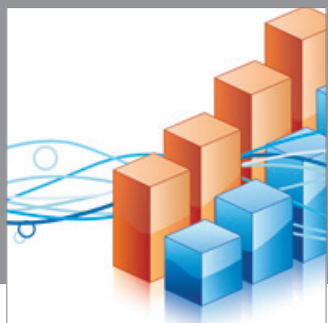

Advances in

Operations Research

mansans

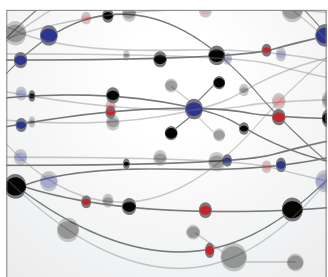

The Scientific World Journal
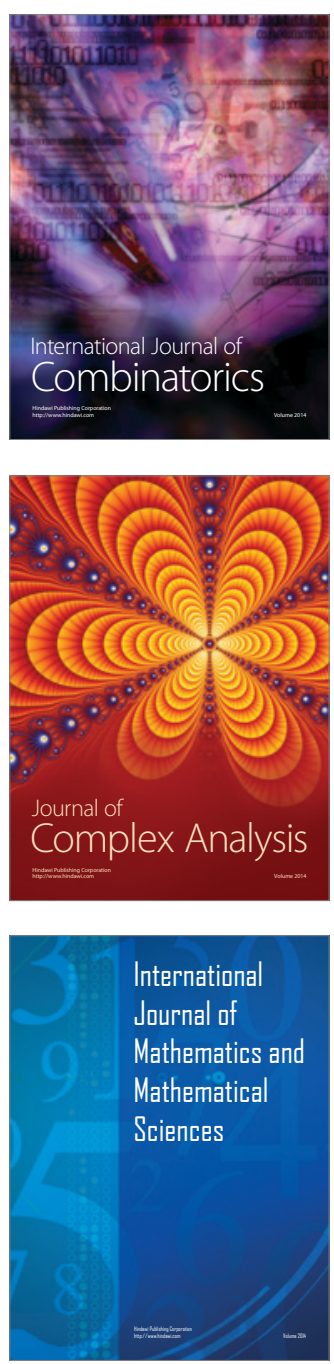
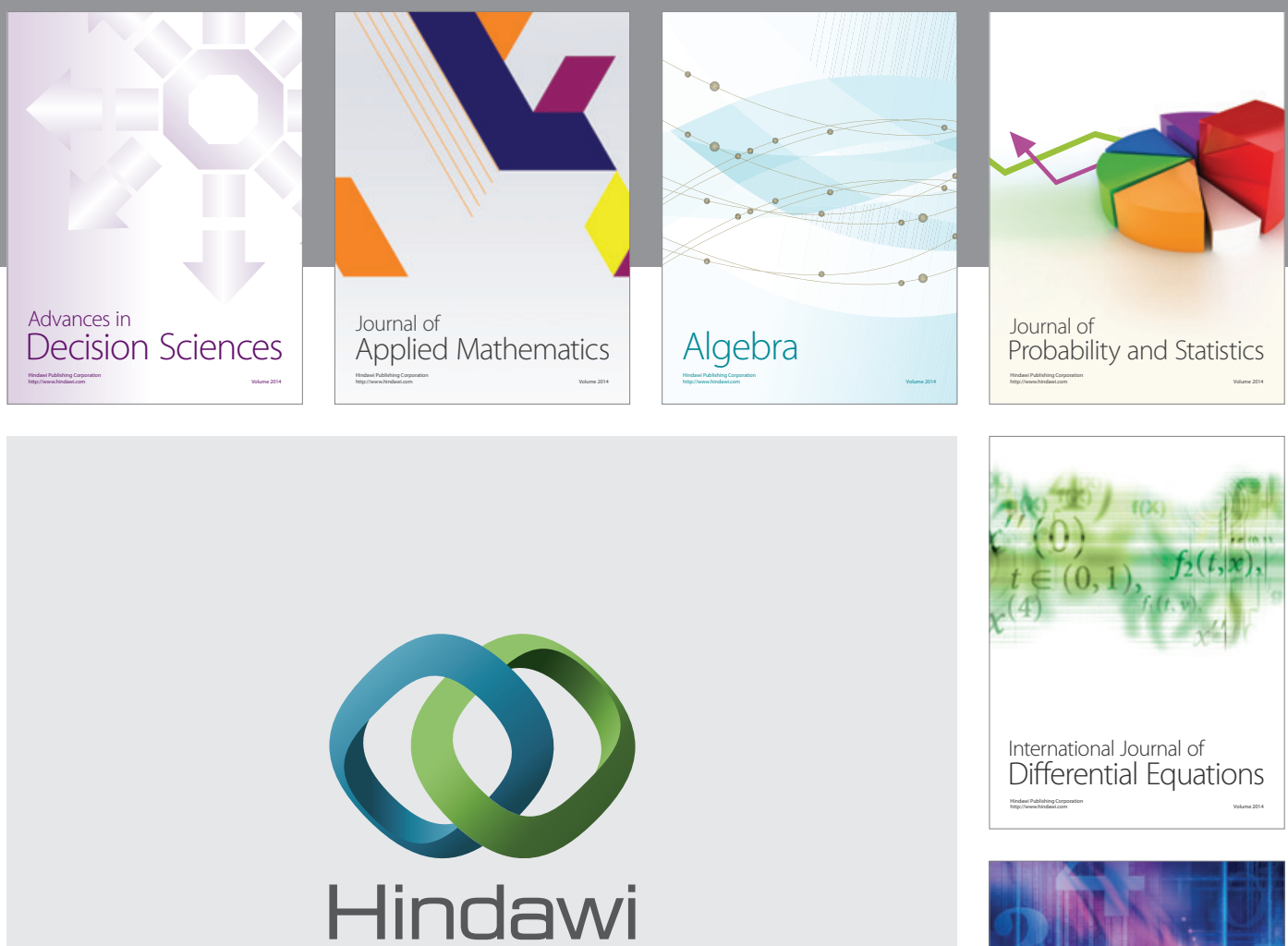

Submit your manuscripts at http://www.hindawi.com
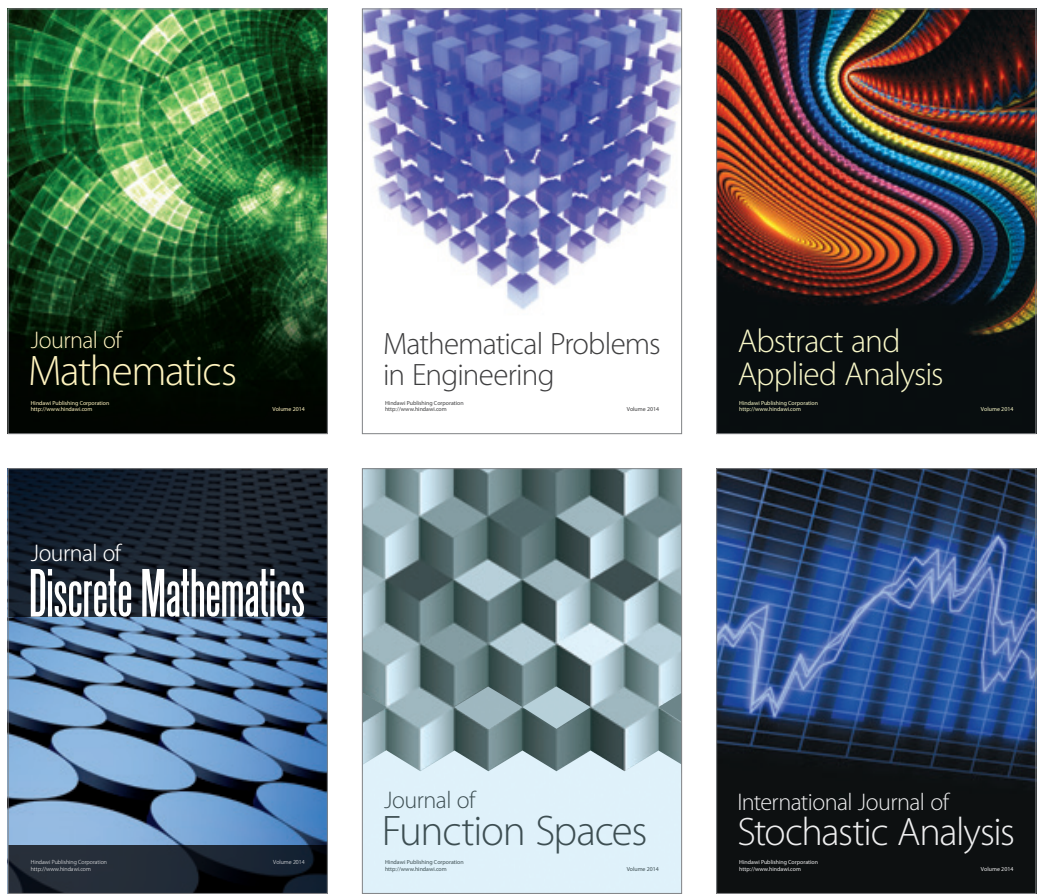

Journal of

Function Spaces

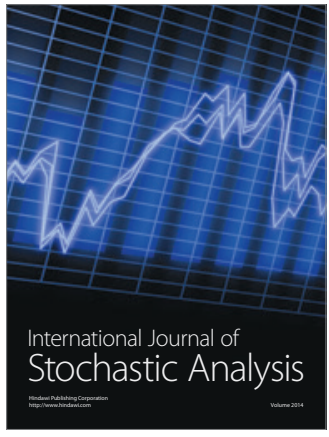

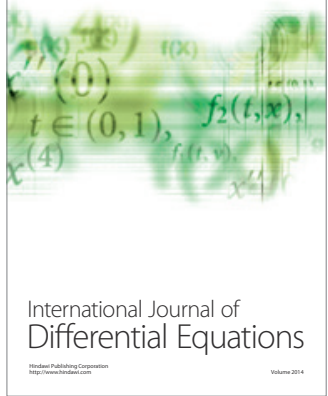
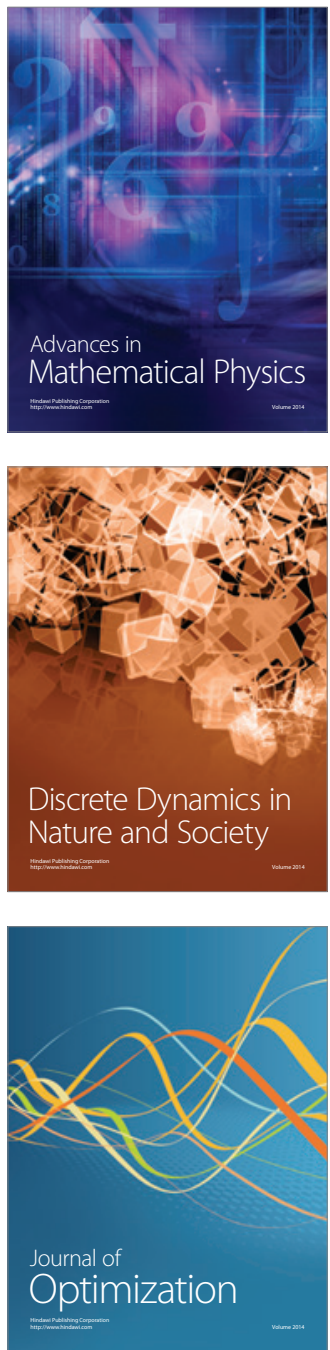